\title{
A influência do soft power na internacionalização dos produtos culturais brasileiros: Uma proposta de framework
}

\author{
Alexandre Rabêlo Neto ${ }^{1 A}$ e José Milton de Sousa-Filho ${ }^{B}$ \\ A Universidade Federal do Piauí - UFPI, Teresina, PI, Brasil. \\ ${ }^{B}$ Universidade Federal de Fortaleza - UNIFOR, Fortaleza, CE, Brasil.
}

\section{DETALHES DO ARTIGO}

\section{Histórico do artigo:}

Recebido em 25 de março de 2015

Aceito em 27 de julho de 2015

Disponível online em 30 de abril de 2016

Sistema de Revisão "Double Blind Review"

Editor científico:

Eduardo Eugênio Spers

\section{Palavras-chaves:}

Produto cultural

Soft power

Internacionalização

\begin{abstract}
RESUMO
A cultura tornou-se um dos setores de mais rápido crescimento nas economias pós-industriais. Conhecer o seu funcionamento, além de ampliar o seu desempenho como fator de ingresso para a economia, nos permitirá agregar a melhoria de condições de vida como parte da mesma estratégia, favorecendo a melhor organização do processo produtivo e acesso aos bens culturais. Internacionalizar significa considerar diferentes níveis de relacionamento e de realização de negócios, desde a compra e venda de produtos e serviços até o investimento em ativos em outros países (investimento direto e investimento de carteira). Desse modo, pode-se incluir nessa discussão o soft power (poder brando) com a finalidade de proporcionar ao país uma possível estratégia de internacionalização, no que se refere aos produtos culturais. O objetivo principal do estudo é analisar a influência do soft power como variável moderadora na internacionalização de produtos culturais brasileiros. Percebe-se que o desenvolvimento de modelos explicativos sobre a internacionalização dos produtos culturais brasileiros é pouco explorado. Portanto, a proposta de framework aqui apresentada, relaciona as variáveis "made in", preferência e internacionalização do produto cultural brasileiro, tendo como variável moderadora o soft power, objetivando oportunizar novas discussões sobre o assunto, além de possibilitar sua aplicação para futuros estudos.
\end{abstract}

(C) 2016 Internext | ESPM. Todos os direitos reservados!

\section{Introdução}

A cultura tornou-se um dos setores de mais rápido crescimento nas economias pós-industriais. Conhecer o seu funcionamento amplia o seu desempenho como um fator da economia permitindo a melhoria de condições de vida como estratégia, favorecendo uma melhor organização do processo produtivo e acesso aos bens culturais (Werthein, 2003). Diante disso, trabalhar o desenvolvimento dos negócios ligados a esse setor, significaria, também, criar um ambiente favorável para o desenvolvimento e internacionalização desses negócios.

Segundo Mariotto (2007), internacionalizar significa considerar diferentes níveis de relacionamento e de realização de negócios, desde a compra e venda de produtos e serviços (comércio exterior) até o investimento em ativos em outros países (investimento direto e investimento de carteira). Kogut e Singh (1988) destacam o aspecto cultural como um dos fatores que devem ser avaliados na seleção da modalidade de internacionalização mediante dois aspectos: a distância cultural entre os países (distância do poder, tentativa de diminuição de incerteza em nível individual, masculinidade/feminilidade e individualismo), e a tentativa de diminuição da incerteza em nível corporativo (aspectos organizacionais e gerenciais de diminuição de risco). 
A Economia da Cultura é hoje um dos setores de maior dinamismo na economia mundial, registrando um crescimento de $6,3 \%$ ao ano, enquanto o conjunto da economia cresce a 5,7\%, integrando o segmento de serviços e lazer, cuja projeção de crescimento é superior à de qualquer outro, com estimativa de crescimento de $10 \%$ ao ano na próxima década (Manso, 2013). Esse potencial de crescimento é bastante elástico, pois o setor depende pouco de recursos esgotáveis, já que seu insumo básico é a criação artística ou intelectual e a inovação (IBGE, 2006).

O processo de globalização e as mudanças tecnológicas aplicadas ao setor cultural modicaram as regras do jogo para artistas e criadores, apresentando ainda com mais força a eterna pergunta de saber como é possível conciliar a criatividade pura com as difíceis realidades econômicas. No setor da música, por exemplo, a assimetria dos fluxos culturais encorajou os artistas locais a explorar o seu talento criativo num mercado cada vez mais mundial, acentuando dessa maneira os processos de aculturação no mundo inteiro (UNESCO, 2009). Diante disso, as escolhas restritas das representações que propõem as grandes redes de mídia e de comunicação tendem a favorecer a criação de estereótipos, manifestadas pela propensão específica para reduzir ou simplificar as coisas, mediante formatações de conteúdos uniformizados. Diante dessa perspectiva, o processo de intercâmbio de informações entre culturas distintas e a consequente absorção de conteúdos simbólicos por meio da aculturação (Cirese, 1979), pode ser entendido como uma forma de soft power, objetivando proporcionar, ao país, uma possível estratégia de internacionalização no que se refere aos produtos culturais.

Embora o "soft power" seja retratado em diversas dimensões, as teorias de Relações Internacionais nos permitem identificar três categorias gerais de poder: econômica, militar,e ideológica e cultural.Todas três são, sem dúvida, importantes, mas a última é muitas vezes negligenciada por alguns políticos. O soft power corresponde à capacidade de conseguir o mesmo objetivo por meio do consentimento. Em outras palavras, pela capacidade de convencer os outros a fazer algo por meio da influência cultural e ideológica (Nye Jr. 2004, 2011). Assim, a atuação da produção cultural, por exemplo no cinema, dado o seu grande potencial de alcance e audiência, pode ser vista como um instrumento de poder ideológico. Nesse contexto, o cinema não é apenas uma imagem ou ilustração da realidade, ele desempenha um papel ativo na formação de identidades e realidades internacionais (Zismann e Geisler, 2013).

A cultura captura o soft power, que tem o poder ideológico de unir a sociedade (Ferreira, 2010), por exemplo, parando uma fábrica multinacional para assistir a Copa do Mundo de Futebol. Na copa do mundo do México, o ônibus da Seleção Brasileira tinha a seguinte inscrição: "Veículo rastreado por 180 milhões de brasileiros", o que realmente acontecia. A prática cultural capturava a hegemonia naquele período (Amphilo, 2012). A Coca-Cola que tem seu rótulo de embalagem vermelho em todo mundo, teve que levar em consideração as características locais da cidade de Parintins, no Amazonas, onde o consumidor filtrava a informação e decidia-se por outra marca, a partir do costume local. Assim, os seguidores do Boi "caprichoso", de cor azul, não consumiam Coca-Cola por causa da cor da embalagem cuja cor representava o Boi "garantido" e se apegavam à Pepsi que tem a embalagem azul. Esse exemplo, demonstra que a cultura local pode ser uma fonte de soft power (Amphilo, 2012; Nye Jr, 2004).

Diante da globalização, as possibilidades de relacionamento entre empresas e produtos culturais, e o uso estratégico destes por aquelas ganha dimensões maiores. Nesse contexto, os produtos culturais ficam mais importantes pelo fato de esses produtos representarem ou serem representados por empresas que, em muitos casos, têm poderes comparados aos de um Estado (Bird; Stevens, 2003); de se ampliarem as possibilidades de empresas agregarem valor capital e fiscal ao investirem ou patrocinarem esses produtos (Becker, 2003); e de as empresas terem descoberto a aplicação do caráter político-simbólico de produtos culturais em suas estratégias corporativas (Alvesson 1991; Mendonça e Amantino-De-Andrade, 2003), também explicado por abordagens na área de estratégia que enfatizam questões de poder (Mintzberg et al, 2000; Arnold, 2003; Martin, 2003).

O Brasil exportou um total de 2.967,9 bilhões de dólares em 2006 no que diz respeito aos seus produtos culturais, Colômbia 195,3 milhões, Argentina 164,9 milhões, Chile 29,1milhões, Peru 21,9 milhões e o Uruguai 15,8 milhões (SICSUR, 2011). O mercado interno brasileiro tem uma produção cultural nacional superior à estrangeira, em que o predomínio chega a $80 \%$. A atividade cultural 
mais presente nos municípios é o artesanato (64,3\%), seguida pela dança (56\%), bandas (53\%) e a capoeira (49\%), esta última além da expressiva presença no país é, ao lado da música, um dos segmentos que maiores interesses despertam no exterior. Os festivais apresentam-se como a mais dinâmica forma de difusão cultural no país: 49\% das cidades contam com festival de cultura popular, 39\% com festival de música, 36\% com festival de dança, 26\% com festival de teatro e $10 \%$ com festival de cinema (IBGE, 2006; SICSUR, 2011). Portanto, nota-se que o mercado de produtos culturais brasileiros pode estar em expansão.

Tendo em vista a importância de estudos no que se refere ao consumo de produtos culturais numa perspectiva de ambiente mercadológico internacional, estabeleceu-se o seguinte problema de pesquisa: Qual a influência do soft power na identidade nacional mediante a internacionalização de produtos culturais brasileiros? nas relações internacionais foi cunhado por Nye Jr. (1980). De acordo com essa visão, o poder brando seria a habilidade de um ator persuadir outros a fazer o que ele deseja sem o emprego da força ou coerção, ou seja, sem recorrer ao poder bruto (hard power).

Na análise conceitual de Nye Jr. (2004), em linhas gerais, o poder brando nos remete a um ambiente internacional no qual o multilateralismo, a cooperação e as instituições liberais são predominantes na vertente do internacionalismo liberal. Assim, para Nye (2004), dado que a força não resolve todos os conflitos, há outras maneiras de um dado ator (A) influir no comportamento de outro(s) $(B, C, \ldots)$ para levá-lo(s) a agir (em) de modo a satisfazer (em) os interesses de A. No entanto, a associação geral entre os tipos de comportamentos e certos recursos é forte o suficiente para nos permitir utilizar o atalho útil para a utilização de hard power $e$ soft power, conforme o Figura 1 a seguir.

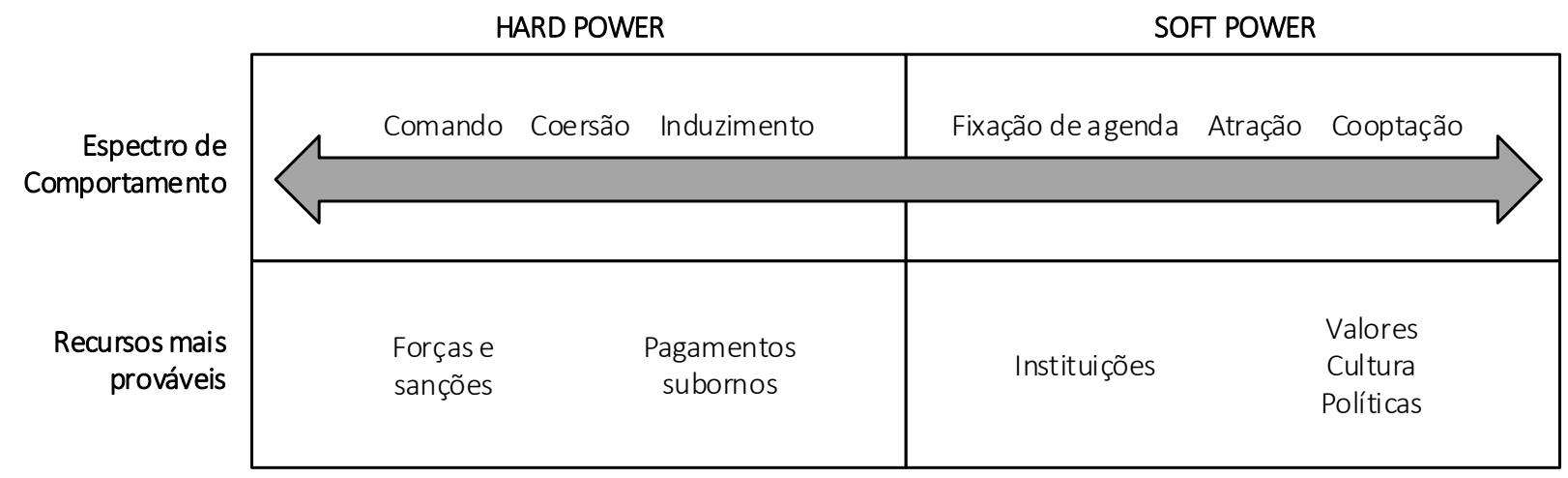

Fig. 1

Associação dos tipos de comportamento e recursos de hard-power e soft power

Fonte: Nye Jr. (2011, p.8) adaptado pelos autores (2014).

Percebe-se que o desenvolvimento de modelos explicativos sobre a internacionalização dos produtos culturais brasileiros é pouco explorado. Assim, esta pergunta origina o objetivo principal do estudo, que é analisar a influência do soft power como variável moderadora na internacionalização de produtos culturais brasileiros. Diante disso, os objetivos específicos para esse estudo são: (a) identificar as variáveis que influenciam na internacionalização de produtos culturais brasileiros; (b) verificar as relações entre soft power e a identidade nacional brasileira.

\section{O soft power e a internacionalização de produtos culturais no mundo}

O emprego da noção de soft power (poder brando) para analisar as ações dos Estados Unidos da América
O soft power de um país reside em três recursos: na sua cultura (atratividade para outros agentes a partir de lugares, eventos, produtos culturais), nos seus valores políticos (a forma de se relacionar com os outros países) e, na sua estratégia de política internacional (pela legitimação ou autoridade moral) (Nye Jr. 2004, 2011).

Inserindo o homem ao ambiente, por meio da cultural (Herskovits, 1955), tem-se que o soft power pode ser associado a muitos produtos e bens de consumo, por exemplo, livros, música, cinema, artesanato, entre outras manifestações populares de massa de arte e educação. Isso fica evidente na forma como os Estados Unidos disseminam sua ideologia pelo mundo a partir de seus produtos culturais (filmes, música, tecnologia, mídia, literatura, livros, 
eventos, valores culturais) e pelo "American way of life", com a finalidade de construir uma persuasão socialmente construída, objetivando uma fonte de recursos importantes onde os grupos sociais poderiam transmitir conhecimentos e valores, em níveis diversos, a partir dos comportamentos sociais.

Nesse sentido, tem-se que a dinamicidade da cultura em si, carece de mais pesquisas voltadas para as relações entre cultura e poder do comportamento (Nye Jr., 2004). Nesse contexto, o cinema permite a disseminação do entendimento de uma nação do que "aconteceu na verdade" historicamente, além do retrato que ela deseja transmitir de si mesma, conforme os três pilares constituintes do soft power, cultura, ideais políticos e políticas. Nota-se que o cinema age em dois âmbitos altamente desejáveis: tanto a construção cultural quanto na disseminação dos ideais políticos do país (Nye Jr., 2004).

A importância da utilização do soft power nas políticas públicas de internacionalização da cultura dos países da América do Sul pode ser evidenciada a partir dos dados do Sistema de Informação Cultural do Mercosul - SICSUR (2011). Os resultados apresentados sugerem que, em 2010, a cultura atingiu 3,5\% do Produto Interno Bruto - PIB na Argentina, e atravessou o seu sexto ano consecutivo de expansão. As atividades culturais mostraram um crescimento sustentável e uma participação estável numa economia nacional que está se expandindo com taxas muito elevadas. O conceito de indústria criativa, evidenciado a partir do desejo de criar coisas cujo valor não seja puramente prático (Demasi, 2003), poderia complementar essa visão.

Segundo Howkins (2007), tanto arte como ciência procuram imaginar (visualizar) e descrever (ou representar) a natureza e o sentido da realidade, utilizando o mesmo procedimento de pensamento e geração de ideias. A diferença está na forma em que se busca imaginar o mundo, e como são resguardados os valores econômicos. Diante disso, o processo criativo é o mesmo, o que os diferencia são os produtos criativos.

Corroborando com essa visão, na Colômbia, o Ministério da Cultura e o Departamento Administrativo Nacional de Estatística - DANE, concretizou um acordo administrativo com a intenção de construir uma aproximação à medição do campo cultural por meio da Conta Satélite de Cultura, identificando atividades produtoras de bens e serviços culturais durante o período de 2000 a 2007. Dentro desta medição, foram incluídas as seguintes atividades: Edição de livros e jornais, Transmissão de Rádio, Televisão e TV à Cabo, Publicidade, Fotografia, Investigação e Desenvolvimento Cultural, Serviços de Divulgação Cultural (que inclui a produção e exibição de Filmes, Rádio, Televisão, Teatro, Serviços de Artísticos, Organizações Particulares de Cultura, entre outros), Museus, Educação Artística e Serviços do Governo, enquanto produzem bens e serviços considerados culturais. A partir dessa mediação, notou-se um crescimento na participação do PIB das atividades culturais dentro da economia total colombiana, passando de uma participação de 1,58\% em 2000 para 1,78\% em 2007. Além disso, o crescimento do PIB das atividades culturais durante o mesmo período foi de $61,39 \%$, ultrapassando o crescimento do PIB de toda a economia do país, que foi 39,38\% (Calcagno et al, 2008).

Mais de 70\% dos 13 mil editores da região estão localizados na Argentina, Brasil e Colômbia. O Brasil domina o mercado de língua portuguesa, enquanto Argentina e Colômbia se impõem nas regiões de língua espanhola. A Espanha desempenha um papel importante para completar a curva de oferta que atende as necessidades locais de livros, ao cobrir 30\% das importações da região. Devido à estrutura do mercado regional, caracterizado por rotas de distribuição de benefícios fiscais e tarifários, Argentina, Colômbia e Chile são usados pelos editores espanhóis como base para a reexportação de livros pela América do Sul. Nos anos recentes, esses três países têm 30\% de importações de livros de origem espanhola, com destaque à Argentina, aonde o número chega a 50\% (Calcagno et al, 2008).

Tab. 1

PIB do núcleo criativo e participação no PIB, países selecionados $-2011$

\begin{tabular}{rcc}
\multicolumn{1}{c}{ País } & $\begin{array}{c}\text { PIB Criativo } \\
\text { (R\$ Bilhões) }\end{array}$ & $\begin{array}{c}\text { Participação } \\
\text { no PIB }\end{array}$ \\
\hline Estados Unidos & 1,011 & 3.3 \\
Reino Unido & 286 & 5.8 \\
França & 191 & 3.4 \\
Alemanha & 181 & 3.2 \\
Brasil & 110 & 2.7 \\
Itália & 102 & 2.3 \\
Espanha & 70 & 2.3 \\
Holanda & 46 & 2.7 \\
Noruega & 32 & 3.2 \\
Bélgica & 27 & 2.6 \\
Suécia & 26 & 2.4 \\
Dinamarca & 21 & 3.1 \\
Áustria & 15 & 1.8 \\
Grécia & 6 & 1.0 \\
\hline
\end{tabular}

Fonte: FIRJAN/UNCTAD com base nos dados do PIB (2011) adaptado pelos autores (2013). 
Em 2011, aproximadamente 243 mil empresas formavam o núcleo da indústria criativa no Brasil (Pinto et al., 2013). Com base na massa salarial dessas empresas, estima-se que o núcleo criativo gera um Produto Interno Bruto equivalente a R\$ 110 bilhões, ou $2,7 \%$ de tudo o que é produzido no País. Esses resultados colocam o Brasil entre os maiores produtores de criatividade do mundo, superando Espanha, Itália e Holanda, conforme a tabela 1.

Retomando a visão de Nye Jr (2011), tem-se que o caso paradigmático dos EUA já não requer ação estratégica do Estado, pode-se citar o exemplo da Inglaterra, que conta com um ministério das indústrias criativas, independente do Ministério da Cultura, como marco da crescente importância que o setor vem adquirindo nas economias nacionais. Do mesmo modo, já começam a se estabelecer programas em estados e, mesmo, em municípios, que identificaram vocações locais capazes de gerar dinâmica econômica. No intuito de fortalecer seu soft power, a China estabeleceu casas de comércio para facilitar os negócios e aumentar os contatos na Ásia Central. A Rádio Internacional da China também está prestando maior atenção à região, agora transmitindo 24 horas por dia. Transmissões para o Cazaquistão, em especial, aumentaram em tamanho e qualidade nos últimos anos, com programas direcionados a ganhar os corações e mentes da população cazaque (Struye, 2010). A esse respeito, como alguns observadores observaram, "se hoje jovens ocidentais vestem roupas chinesas e brincam com brinquedos chineses, não é de surpreender que seus filhos e netos um dia vão preferir ouvir música pop chinesa e ver filmes produzidos na China" (Tremblay, 2007).

O soft power, no caso do Japão, pode ser exemplificado pela internacionalização das revistas em quadrinhos ou Mangás, que dotados de uma rica variedade de gêneros e subgêneros, tornaram-se acessíveis a um público amplo, constituído desde crianças em processo de alfabetização até adultos de ambos os sexos. Criado no Japão a partir de expressões artísticas autóctones e estrangeiras, o mangá ficou restrito ao arquipélago asiático por décadas. Cenário bem diferente do final do século XX e início de século XXI, quando o mangá deixa de ser apenas japonês para se desterritorializar, virar uma mobília da modernidade-mundo: sua influência vai estar agora nas histórias em quadrinhos do Ocidente, nos eventos de cultura pop japonesa, nos concursos de novos autores estrangeiros de mangá, na publicidade e na paisagem urbana (Hirata, 2012). Como exemplos, tem-se a inserção de desenhos no estilo mangá em isqueiros colecionáveis da marca Bic vendidos no Brasil; o uso da personagem Hatsune Miku no comercial do Toyota Corolla vendido nos Estados Unidos; a criação da Turma da Mônica Jovem pelo desenhista Maurício de Souza, cujos personagens e historias emulam muito das características do mangá; a grafitagem na cidade de São Paulo, no bairro da Liberdade; e as convenções brasileiras e internacionais de fãs de mangás (Hirata, 2012).

No que diz respeito ao Brasil, a facilidade de absorção de novas tecnologias; a criatividade e a vocação para inovação; a disponibilidade de profissionais de alto nível em todos os segmentos da produção cultural e; a alta qualidade e a boa aceitação de nossos produtos culturais em diferentes mercados podem ser vistos como aspectos de diferenciação do país. Outro ponto importante é que o mercado interno brasileiro é muito expressivo, com uma produção cultural nacional superior à estrangeira, por exemplo, a música e o conteúdo de TV, em que o predomínio chega a $80 \%$. A atividade cultural mais presente nos municípios é o artesanato $(64,3 \%)$, seguida pela dança (56\%), bandas (53\%) e a capoeira (49\%).

Os festivais apresentam-se como a mais dinâmica forma de difusão cultural no país: $49 \%$ das cidades contam com festival de cultura popular, 39\% com festival de música, 36\% com festival de dança, 26\% com festival de teatro e $10 \%$ com festival de cinema (IBGE, 2006). Até 2020, o Ministério da Cultura do Brasil deverá investir US\$ 35 milhões em ações que vão desde o financiamento da tradução e edição de obras nacionais até programas de intercâmbio de autores e tradutores e participação nas principais feiras do mundo. A nova política brasileira na área internacional compreende três diferentes frentes. A primeira busca ampliar a presença dos livros brasileiros em diferentes idiomas e países do mundo. A segunda é dirigida basicamente aos países da América Latina que falam espanhol. E a terceira compreende os próprios países que falam português na Europa, África e Ásia, por meio da criação de um edital específico para apoiar a publicação de obras brasileiras pelas editoras desses países. Isso demonstra que o governo brasileiro busca o soft power como forma de internacionalização da literatura (MINC, 2012). 


\section{Proposições teóricas do estudo e modelo teórico}

As proposições deste estudo versam sobre as relações que podem existir entre o fator "made in" e a preferência por produtos culturais brasileiros, e também, a possível influência existente da preferência por produtos culturais brasileiros no que se refere à internacionalização desses produtos. Diante disso, buscou-se na literatura um aprofundamento do conhecimento sobre essas perspectivas com o objetivo de propor um modelo teórico que contemplasse todos esses constructos. A variável soft power, no modelo teórico, foi incluída como um fator moderador dos relacionamentos entre os constructos da pesquisa.

O fator "made in", como forma de internacionalização, é uma das áreas que mais podem beneficiar o crescimento econômico e a competitividade nacionais. Nesse sentido, quanto mais favorável for a imagem de um país, mais proeminente será a promoção dos seus produtos, e vice-versa (Bradley, 1995). Considerando as exportações, a principal influência de uma marca nacional forte advém do valor acrescentado que atribui às empresas, produtos e serviços que surgem associadas à sua nacionalidade. Neste sentido, diversos estudos procuram avaliar o impacto do "made in" nas percepções de qualidade nos consumidores demonstrando o peso do selo nacional em produtos (Phau e Prendergast, 2000; Kotler e Gertner, 2002).

Por outro lado, existe uma relação entre as preferências dos consumidores por produtos de um país e a percepção da cultura, economia e políticas desse mesmo país (Mossberg e Kleppe, 2005), no que se refere à imagem da marca é, ao nível do território, um possível reflexo da sua personalidade (Rainisto, 2007). Na verdade, tal como um país, uma cultura ou um povo, as marcas possuem um valor emocional e social para os consumidores (Kotler e Gertner, 2002). Se hoje claramente se aceita a ideia de que as marcas atribuem ou subtraem valor ao produto, a relação que se estabelece com o país de origem é fundamental na avaliação do valor. Neste sentido, a cultura apresenta-se como um meio e um fim no marketing territorial, realçando que existem sinais de profundidade cultural no processo de desenvolvimento econômico de cidades e regiões (Campbell, 2007).

Outra visão a respeito da influência do país de origem na avaliação de um produto encontra respaldo no modelo de Fishbein e Ajzen (1975). Segundo essa visão, a imagem do país afeta diretamente as crenças acerca dos atributos dos produtos, os quais influenciam a atitude geral para com os produtos. Portanto, o país de origem afeta de forma indireta as atitudes por meio das crenças, fenômeno geralmente descrito como efeito halo (Ayrosa, 2000). Diante disso, no consumo cultural a racionalidade perderia força, já que os gostos e preferências não são estáveis e sim, evoluem com o tempo. Nessa perspectiva, explica-se o fato do prazer e da vontade de consumir aumentarem proporcionalmente ao consumo. Assim, percebe-se que os gostos e preferências evoluem com o tempo, contrariando o princípio da racionalidade (Benhamou, 2007, p. 30).

O efeito "país de origem" é indicado para a descrição da influência do país de origem nas atitudes, percepções de produto e comportamento do consumidor. Essa influência é determinada por sentimentos relacionados ao país e a estimativas racionais da qualidade do país, associados por intermédio da imagem expressa pela etiqueta "made in". Segundo Kotler (2000), imagem e identidade são conceitos diferentes. A identidade está relacionada à identificação e posicionamento de bens de consumo. Imagem é a maneira como o público vê a empresa ou seus produtos. Uma imagem efetiva precisa estabelecer a personalidade do produto e a proposta de valor; deve transmitir essa personalidade de maneira distinta, para que não seja confundida com a dos concorrentes; deve comunicar um poder emocional que vai além de uma simples imagem mental. Conforme Ger (1999), qualquer categoria de produto pode incorporar a cultura local. Desse modo, diferentes categorias de produtos carregam a identidade cultural de seu país de origem, interpretada pelos compradores no momento da aquisição.

Diante disso, a proposição que busca relacionar o fator "made in" e a preferência por produtos culturais brasileiros é:

P1: O fator "made in" influencia positivamente na preferência por produtos culturais brasileiros.

Uma expressão utilizada na literatura para significar o efeito do consumo passado sobre o presente é a ideia de "learning by consuming". Segundo essa visão, o indivíduo não saberia qual é o seu gosto e só o descobre mediante repetidas experiências de 
consumo (DINIZ, 2009). Cada novo experimento revela um aumento ou uma redução do gosto. Essa abordagem baseia-se na ideia das expectativas racionais, enquanto a ideia de "vício racional" ancorase na hipótese de comportamento racional maximizador dos agentes, embora a essência do argumento seja igual para ambas (Lévy-Garbona e Montmarquette, 2003).

Os gostos, assim como as necessidades, variam conforme o nível econômico. Enquanto as classes mais baixas têm como estilo de vida a realização das necessidades básicas do mundo, as classes mais abastadas, que possuem melhores condições de satisfazer essas prioridades, buscam necessidades que, para os menos favorecidos, são luxos irrealizáveis. Esses luxos são objetos de conforto que se tornam necessidade. As práticas realizadas nessas condições se constituem "numa experiência liberada da urgência e na prática de atividades que tenham nelas mesmas sua finalidade" (Bourdieu, 1983, p.87).

Por outro lado, atributos culturais específicos podem beneficiar a posição de um país no que tange ao comércio internacional. Levchenko (2004) e Nunn (2007) constataram que boas instituições são fonte de vantagem comparativa no comércio internacional para os setores onde os efeitos de tais instituições são mais relevantes. Assim sendo, as afinidades culturais entre países podem motivar o comércio internacional. Segundo Spolaore e Wacziarg (2013), a própria cultura origina vários mercados, nomeadamente por meio do comércio cultural internacional. Nessa conjuntura, o comércio cultural pode abrir mercados quer diretamente, pelo seu próprio desenvolvimento, quer indiretamente, pela aproximação das culturas dos países com o objetivo de estimular o comércio bilateral.

Hermans e Kempen (1998) examinaram o processo de internacionalização e a interligação de culturas em todo o globo. Eles demonstraram que a cultura é cada vez mais deterritorilizada e não é necesesariamente limitada a locais específicos. Pieterse (2009) analisou a cultura global em termos de hibridação da cultura. Nesse sentido, novas formas de cultura estão sendo criadas pela combinação de elementos de duas culturas existentes motivando outras possibilidades de preferências pelos produtos culturais dos países por meio da hibridação. Rowe e Schelling (1991) dão exemplos de hibridização, como o rap asiático, os bagels Irlandeses, os tacos chineses, Mardi Gras indianas, e as meninas das escolas mexicanas vestidas com togas gregas dançando no estilo de Isidora Duncan. Essas mudanças se ampliam nas cidades pela proximidade física entre os indivíduos de diversas origens culturais. Portanto, pressupõe-se que a preferência influencia a internacionalização, no que se refere aos produtos culturais brasileiros. Diante disso, a segunda proposição do estudo é:

P2: A preferência influencia positivamente a internacionalização do produto cultural brasileiro.

Tendo em vista que o soft power de um país pode residir na sua cultura (atratividade para outros agentes a partir de lugares, eventos, produtos culturais), nos seus valores políticos (a forma de se relacionar com os outros países) e, na sua estratégia de política internacional (pela legitimação ou autoridade moral) (Nye Jr. 2004, 2011), nota-se essa variável pode funcionar como um fator moderador nos relacionamentos existentes entre: fator "Made in", preferência por produtos culturais brasileiros e a internacionalização de produtos culturais brasileiros. Portanto, percebe-se que a internacionalização, nesse estudo, busca uma perspectiva relacional, no que se refere às redes de relacionamento (Johanson e Mattson, 1998; Teixeira e Diz, 2005), a partir das diferentes respostas face às oportunidades que emergem nos mercados internacionais resultantes das crenças da gestão (Dias, 2005). Nesse contexto, os níveis de envolvimento das empresas do setor cultural com as estratégias de marketing internacional pode se caracterizar como um possível policentrismo, que seriam estratégias de marketing adaptada a cada país, com produtos ou serviços focados no mercado alvo (Dias, 2005).

O processo de descentralização cultural, faz com que as culturas locais ganhem, reconquistem espaços, dentro da cultura nacional. Hoje, a necessidade de integração nacional, coexiste cada vez mais com a necessidade de abertura e de visibilidade pelo mundo afora, mediante promoção turística, por exemplo. Isso pode ser traduzido em atratividade, em vontades de criar ou manter um poder de atração ou de influência indireta, ou soft power, por meios culturais ou ideológicos, pelo mundo afora. Este "mundo afora" do Estado-Nação pode ser definido como o espaço da circulação global, dentro do qual as nações interagem e competem. Assim, diante da globalização, já não se trata de um grupo ou de uma comunidade migrante fechada, 
"mantendo a tradição" entre si, só entre si, mas sim de um potencial de visibilidade considerável, facilitado pelos meios de comunicação modernos, consequentemente, de efeitos indiretos de atração de soft power. Por exemplo, as bandas brasileiras instaladas e, sobretudo, reconstituídas em Nova Iorque (os Nation Beat, os Maracatu New York, os Forró in the Dark, a associação Samba Nation), têm um papel determinante na criação de uma música brasileira "autêntica" made in New York (La Barre, 2010). Diante disso, a cultura "gera valor" pela incorporação de formas de vida aos produtos: estilos, preferências, status, subjetividades, informações, normas de consumo. Assim, a mercadoria precisa ser dotada de valor cultural. Portanto, a terceira proposição da pesquisa apresenta a variável soft power como fator de moderação no relacionamento entre fator "Made in" e preferência e, a quarta proposição do estudo busca a relação entre preferência e a internacionalização de produtos culturais brasileiros.

P3: A variável soft power influencia positivamente o relacionamento entre o fator "made in" e a preferência por produtos culturais brasileiros.

P4: A variável soft power influencia positivamente o relacionamento entre a preferência e a internacionalização de produtos culturais brasileiros.

Na figura 2, apresenta-se o modelo proposto para o estudo.

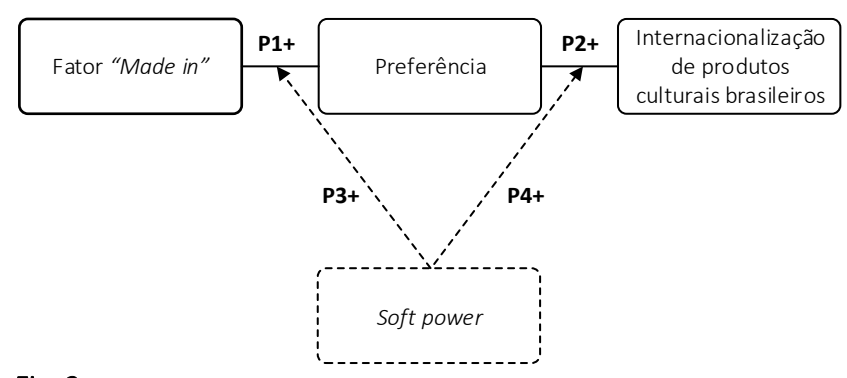

Fig. 2

Modelo proposto para o estudo

Fonte: Próprios autores (2014)

\section{Considerações finais}

A reflexão sobre o consumo de produtos culturais numa perspectiva de ambiente mercadológico internacional embasou o problema dessa pesquisa, onde se buscou entender qual a influência do soft power na internacionalização dos produtos culturais brasileiros. Em contrapartida, visto que o desenvolvimento de modelos explicativos sobre a internacionalização dos produtos culturais brasileiros, ainda são pouco explorados, o objetivo principal do estudo foi analisar a influência do soft power como variável moderadora na internacionalização de produtos culturais brasileiros.

Diante disso, buscou-se na literatura um aporte teórico no que se refere a soft power (Nye Jr., 2004, 2011; Calcagno Et Al, 2008; Hirata, 2012; Struye, 2010; Tremblay, 2007;), ao fator "made in" (Bradley, 1995; Phau e Prendergast, 2000; Kotler e Gertner, 2002; Ger, 1999; Rainisto, 2007), a preferência (LévyGarbona e Montmarquette, 2003; Bourdieu, 1983; Pieterse, 2009; Diniz, 2009) e, a internacionalização de produtos culturais (Demasi, 2003; Calcagno et al, 2008; Hirata, 2012; Struye, 2010; Tremblay, 2007; Dias, 2005)

As proposições discutidas nessa pesquisa foram: P1 - O fator "made in" influencia positivamente na preferência por produtos culturais brasileiros; P2 - A preferência influencia positivamente a internacionalização do produto cultural brasileiro; P3 - A variável soft power influencia positivamente o relacionamento entre o fator "made in" e a preferência por produtos culturais brasileiros; P4 - A variável soft power influencia positivamente o relacionamento entre a preferência e a internacionalização de produtos culturais brasileiros.

Essas proposições teóricas precisam ser testadas empiricamente em futuros estudos. Portanto, recomenda-se que pesquisas futuras busquem testar as proposições surgidas a partir desse estudo, com a finalidade de ampliar o entendimento sobre os relacionamentos encontrados, a partir da literatura, no que tange à preferência por produtos culturais, ao fator "made in" e a internacionalização de produtos culturais, tendo como fator moderador ou mediador a variável soft power.

\section{Referências}

- AJZEN, I.; FISHBEIN, M. (1975) Understanding attitudes and predicting social behaviour. New Jersey: Prentice-Hall.

- ALVESSON, M. (1991) Organizational Simbolism and Ideology. Journal of Management Studies, 28 (3), p. 207-225. DOI: 10.1111/j.14676486.1991.tb00945.x

- AMPHILO, M. I. (2012) Folkcomunicação e as Teorias Sociais. RAZÓN Y PALABRA Revista 
Electrónica en América Latina Especializada en Comunicación. 80, 1-23.

- $\quad$ ANHOLT, S. (2003) Brand New Justice: The Upside of Global Branding, Oxford: ButterworthHeinemann.

- AYROSA, E. (2000) Some notes on the development of research on country-of-origin effects. Arché Internacional, Rio de Janeiro, ano IX (26).

- ARNOLD, D. (2003) Libertarian Theories of the Corporation and Global Capitalism. Journal of Business Ethics, 43 (2), p.155-173.

- BAIARDI, A. (1996) Revista Baiana de Tecnologia TECBAHIA. Ciência, Tecnologia e a Rediscussão da Questão Regional. Camaçari. 11 (1) , .

- BECKER, I. A. (2003) Captação de Recursos: Planejamento, Prática e Resultados. Um estudo em Museus do Brasil. (Tese de Doutorado) - Fundação Getúlio Vargas - EAESP. BRA.

- Benhamou, F. (2007) A economia da cultura. Trad. Geraldo Gérson de Souza. São Paulo: Ateliê Editorial.

- BIRD, A. \& STEVENS, M. (2003) Toward an Emergent Global Culture and the Effects of Globalization on Obsolescing National Cultures. Journal of International Management. 9(4), p.395407. DOI:10.1016/j.intman.2003.08.003

- BourdieU, P. (1984) La Distinction. London, England: Routledge.

- BOURDieU, P. (1983) Gostos de classe e estilos de vida. In ORTIZ, R. (org). Pierre Bourdieu: Sociologia. pag. 82-121. São Paulo: Ática.

- BRADLEY, F. (1995) International Marketing Strategy, 2a ed. London: Prentice-Hall.

- CAMPBELL, L. (2007) Community Culture, Local Economic Development and Regional Branding in Ontario, Canada, Regions Magazine, 268(1),17-18. DOI:10.1080/13673882.2007.8628827

- CIRESE. M. (1979) Cultura hegemónica y culturas subalternas. Traducción y organización de Dr. Manuel Velázquez Mejía, México: Universidad Autónoma Del Estado de México.

- Cunha, G. (2011) Artesanato: questões da comercialização. Portugal: CEARTE.

- DEMASI, D. (2003) Criatividade e Grupos Criativos. Rio de Janeiro: Sextante.

- DIAS, Á. L. (2005) Princípios de marketing internacional - Campeões portugueses no estrangeiro. Lisboa: Lidel - Edições Técnicas.
- DINIZ, S. (2009) Análise do consumo de bens e serviços artístico-culturais no Brasil metropolitano. (Dissertação de Mestrado) - Universidade Federal de Minas Gerais-UFMG, BRA.

- DOSI, G. SOETE, L. (1990) Technical change and international trade. In Dosi, G. et al. Technical change end economic theory. London: Pinter Publishers.

- FERREIRA. M. A. F. S. (2014) Definições conceituais para o entendimento da política externa dos Estados Unidos: as noções de poder duro (hard power) e poder brando (soft power). Disponível em:

http://www.santiagodantassp.locaweb.com.br/br /arquivos/nucleos/artigos/Marcos.pdf. Acesso em. 23 de maio de 2014.

- GER, G. (1999) Localizing in the global village: local firms competing in global markets. Califórnia Management Review, v. 41(4), p. 64-83. DOI: $10.2307 / 41166010$

- HERMANS, H. J. M; KEMPEN, H. J. G. (1998) Moving cultures: The perilous problems of cultural dichotomies in a globalizing society. American Psychologist, 53(10), p. 1111-1120. DOI: http://dx.doi.org/10.1037/0003-066X.53.10.1111

- HERSKOVITS, M. J. Cultural Anthropology: An Abridged Revision of Man and His Works. New York, Alfred A. Knopf, Inc. xvi, xxxiv + 569 pp. 1955.

- HiRATA, T. (2012) Mangá: Do Japão Ao Mundo Pela Prática Midiática Do Scanlation. (Dissertação de Mestrado) Universidade Federal de Mato GrossoUFMT, BRA.

- jOHANSON, J.; MATTSSON, L.G. (1998) Internationalization in industrial systems - a network approach. In N. Hood \& J-E. Vahlne (eds). Strategies in Global Competition. New York: Croom Helm. DOI: 10.1057/9781137508829_5

- HOWKINS, J. (2007) The Creative Economy - How People Make Money from Ideas. Londres: Penguin Books.

- iBge - INSTITUto bRAsileiRo de geOgRAFIA E ESTATÍsTICA (2006). Pesquisa de Informações Básicas Municipais - Cultura. Disponível em: <http://www.ibge.gov.br/home/estatistica/econo mia/perfilmunic/cultura2006/cultura2006.pdf> Acesso em: 10 junho. 2014.

- KOGUT, B. \& SINGH, H. (1988) The effect of national culture on the choice of entry mode. Journal of International Business Studies, 19 (3), p. 411. 
- KOTLER, P; GERTNER, D. (2002) Country as Brand, Product and Beyond: A Place Marketing and Brand Management Perspective. Brand Management, 9 (4-5). Disponível em: http://www.ingentaconnect.com/content/pal/bm /2002/00000009/F0020004/art00003. Acesso em. 19/06/2014.

DOI: http://dx.doi.org/10.1057/palgrave.bm.2540076

- KOTLER, P. (2000) Administração de Marketing: a edição do novo milênio. 10. ed. São Paulo: Prentice Hall.

- LA BARRE, J. (2010) Música, cidade, etnicidade: explorando cenas musicais em Lisboa. In CORTEREAL. Maria de São José (org). Revista Migrações Número Temático Música e Migração, n.07, Lisboa. ACIDI, Outubro. p.147-166.

- LEVCHENKo, A. (2004) Institutional Quality and International Trade. IMF Working Paper, DOI: 10.1111/j.1467-937X.2007.00435.X

- lÉVy-garbona, l. \& montmarquette, C. (1995) Comportament dês ménages: me étude économetrique de la demande de théátre sur données in dividuelles. Économie et Prevision. no 121, MANSO, J. R. P. (2014) A Economia da Cultura: Vetor Estratégico de Desenvolvimento para Portugal. Revista Online do Museu de Lanifícios da Universidade da Beira Interior. Disponível em: http://www.ubimuseum.ubi.pt/n02/docs/ubimus eum02/ubimuseum02.jose-pires-mansoeconomia-cultura.pdf. Acesso em. 19/06/2014.

- MARIOTTO, F. L. (2007) Estratégia internacional da empresa. São Paulo: Thomson Learning.

- MENDONÇA, J. R. \& AMANTINO-DE-ANDRADE, J. (2003) Gerenciamento de Impressões: Em Busca de Legitimidade Organizacional. Revista de Administração de Empresas, 43 (1), p. 36-48. DOI: http://dx.doi.org/10.1590/S003475902003000100005

- MINC - MINISTÉRIO DA CULTURA - Diretrizes do Minc para 2012. Disponível em. http://www.cultura.gov.br/documents/10883/12 979/APRESENTACAO_programas-prioritarios2012-site.pdf/7ddc9d80-6537-4284-a171104da2edc2b4. Acesso em. 23/06/2014.

- Mintzberg, H.; AhLSTRAND, B. \& LAMPEL, J. (2000) Safari de Estratégia. Porto Alegre: Bookman.

- MOSsberg, L., E KLEPPE, I. (2005) Country and Destination Image: Different or Similar Image Concepts? The Service Industries Journal, 25 (4), p. 493-503. DOI:10.1080/02642060500092147
- CAlcagno, n. CEnTEno, E. E. C. (2008) Nosotros y los otros: comercio exterior de bienes culturales en América del Sur / Natalia Calcagno y Emma Elinor Cesín Centeno. - 1a ed., p. 152, Buenos Aires: Secretaría de Cultura de la Presidencia de la Nación.

- NUNN, N. (2007) Relationship-Specificity, Incomplete Contracts, and the Pattern of Trade. The Quarterly Journal of Economics, 122 (2) p. 569600.

- NYE Jr, J. S. (2011) The Future of Power. p. 28. New York: Public Affairs.

- NYE Jr, J. S. (2004) Soft Power. 191 p. New York: Public Affairs. DOI: 10.2307/1148580

- PHAU, I. E. \& PREndergast, G. (2000) Conceptualizing the Country of Origin of Brand. Journal of Marketing Communications, 6(3), p. 159-170. DOI:10.1080/13527260050118658

- PIETERSE, J. N. (2009) Globalization and culture: Global mélange. 2. ed. Lanham: Rowman \& Littlefield.

- Pinto, G. B. S. \& AFOnSO, M. F. A. L. O (2013) Mapeamento das Indústrias Criativas no Brasil. Revista de Design, Inovação e Gestão Estratégica REDIGE. 4 (Edição Especial), .

- RAINISTO, S. (Inverno de 2007), "Success Factors of Place Branding" em Revista City and Regional Branding, no 268, Seaford, Regional Studies Association.

DOI:10.1080/13673882.2007.8628829

- UNESCO - RELATÓRIO MUNDIAL DA ORGANIZAÇÃO DAS NAÇÕES UNIDAS PARA EDUCAÇÃO, A CIÊNCIA E A CULTURA (2009) Investir na diversidade cultural e no diálogo intercultural. $40 \mathrm{p}$

- SAMpaio, H. (2003) A experiência do artesanato solidário. In. Políticas culturais para o desenvolvimento: uma base de dados para a cultura. p. 236, Brasília: UNESCO Brasil.

- SISTEMA FIRJAN (2008) A Cadeia da Indústria Criativa no Brasil.

Indústria Criativa no Brasil.

- SICSUR - SISTEMA DE INFORMACIÓN CULTURAL DEL MERCOSUR - ENCLAVE CULTURAL (2011) 1 (1).

- SPOlAORE, E. \& ROMAIN, W. (2013) How Deep Are the Roots of Economic Development? Journal of Economic Literature, 51(2), p. 325-369. (DOI): 10.3386/w18130 
- REINO UNIDO (2011) DCMS - Creative Industries Economic Estimates - Full Statistical Release. Londres.

- ROWE, W. \& SCHELLING, V. (1991) Memory and Modernity: Popular culture in latin America. London: Verso.

- StRuye, T. (2009) Offensive Chinoise en Afrique. Notes d'analyse de la Chaire Inbev Baillet - Latour, Université Catholique de Louvain, 3, april.

- TEIXEIRA, S. \& DIZ, H. (2005) Estratégias de internacionalização. Lisboa: Publisher Team.

- TREMBLAY, M. (2014) L'émergence du soft power chinois. Plateforme Québécoise de Journalisme Citoyen,2007. Disponível em: <http://www.centpapiers.com/lemergence-dusoft-power-chinois/799/>.Acesso. 30 de maio. 2014.

\section{Sobre os Autores}

- Alexandre Rabêlo Neto é Doutorando em Gestão de Empresas pela Universidade de Fortaleza UNIFOR/CE. Também é professor da Universidade Federal do Piauí - UFPI/PI, Brasil. E-mail: alexandrenaka@hotmail.com

- José Milton de Souza-Filho é Doutor em Administração de Empresas pela Fundação Getúlio Vargas EAESP/FGV/SP. Também é professor adjunto da Universidade de Fortaleza - UNIFOR/CE, atuando no Programa de Pós-Graduação em Administração - PPGA, e no Mestrado Profissional em Administração -MPA. E-mail: miltonsousa@unifor.br
- VERNON, R. (1979) La inversion internacional y el comercio internacional em el ciclo de productos. In Rosenberg, N. Economia Del cambio tecnológico. México: Fondo de Cultura Econômica.

- VIANA, C. S. S. (2010) Investimentos Diretos Brasileiros no Exterior em Cenário de Risco Político: Evolução Recente e Impactos na Política Externa. (Dissertação de Mestrado) - Instituto Rio Branco, BRA.

- ZISMANN, T. \& GEISLER, L. (2013) Argo: Uma aventura ocidental em terras orientais. Século XXI. 4 (1), p. 89-104.

- WertThein, J. (2003) Políticas culturais para o desenvolvimento: uma base de dados para a cultura. p. 13, Brasília: UNESCO Brasil. 


\title{
The influence of soft power on the internationalization of Brazilian cultural products: Proposal for a framework
}

\author{
Alexandre Rabêlo Neto ${ }^{A}$ and José Milton de Sousa-Filho ${ }^{B}$ \\ ${ }^{A}$ Federal University of Piauí - UFPI, Teresina, PI, Brazil. \\ ${ }^{B}$ Federal University of Fortaleza - UNIFOR, Fortaleza, CE, Brazil.
}

\begin{tabular}{l}
\hline ARTICLE DETAILS \\
\hline Article history: \\
Received 25 March de 2015 \\
Accepted 27 July 2015 \\
Available online in 30 April 2016 \\
Double Blind Review System \\
Scientific Editors \\
Eduardo Eugênio Spers \\
\hline
\end{tabular}

Keywords:

Cultural product

Soft power

Internationalization

\begin{abstract}
Culture has become one of the fastest growing sectors in post-industrial economies. To know its function and to expand its performance as a factor of inflow in the economy will allow us to improve living conditions, favoring the better organization of productive processes and access to cultural goods. Internationalizing means considering different levels of relationship and business execution, from the buying and selling of products and services to investment in assets in other countries (direct investment and portfolio investment). Soft power is included in this discussion because of its potential to give a country an internationalization strategy with regard to cultural products. The main objective of the study is to analyze the influence of soft power as a moderating variable in the internationalization of Brazilian cultural products. The development of explanatory models on the internationalization of Brazilian cultural products has been little explored. The proposed framework presented here relates to the variables "made in", preference, and internationalization of Brazilian cultural product with the moderating variable of soft power, aiming to provide opportunities for further discussions on the subject, besides enabling its application for future studies.
\end{abstract}

(C) 2016 Internext | ESPM. All rights reserved! 